\title{
A Non-Additive Interaction of a Functional MAO-A VNTR and Testosterone Predicts Antisocial Behavior
}

\author{
Rickard L Sjöberg*,1,2,7, Francesca Ducci ${ }^{2,7}$, Christina S Barr ${ }^{3}$, Timothy K Newman ${ }^{4}$, Liliana Dell'Osso ${ }^{5}$, \\ Matti Virkkunen ${ }^{6}$ and David Goldman ${ }^{2}$ \\ 'Uppsala University Centre for Clinical Research, Central Hospital, Västerås, Sweden; '2 Laboratory of Neurogenetics, NIHINIAAA, Rockville, \\ MD, USA; ${ }^{3}$ Laboratory of Clinical and Translational Studies, NIHINIAAA, Poolesville, MD, USA; ${ }^{4}$ Department of Psychiatry, University of Cape \\ Town, Cape Town, South Africa; ${ }^{5}$ Department of Psychiatry, University of Pisa, Pisa, Italy; ${ }^{6}$ Department of Psychiatry, University of Helsinki School \\ of Medicine, Helsinki, Finland
}

\begin{abstract}
A functional VNTR polymorphism in the promoter of the monoamine oxidase A gene (MAOA-LPR) has previously been shown to be an important predictor of antisocial behavior in men. Testosterone analogues are known to interact with the MAOA promoter in vitro to influence gene transcription as well as in vivo to influence CSF levels of the MAO metabolite 3-methoxy-4-hydroxyphenylglycol (MHPG) in human males. We examined the possible joint effects of testosterone (measured in CSF) and MAOA-LPR genotype on antisocial personality disorder and scores on the Brown-Goodwin Aggression scale in 95 unrelated male criminal alcoholics and 45 controls. The results confirm that MAOA genotype and CSF testosterone interact to predict antisocial behaviors. The MAOA/testosterone interaction also predicted low levels of CSF MHPG, which tentatively suggests the possibility that the interaction may be mediated by a direct effect on gene transcription. If replicated these findings offer plausible explanations for previous inconsistencies in studies of the relationship between testosterone and male human aggression, as well as for how MAOA genotype may influence aggressive behavior in human males. Neuropsychopharmacology (2008) 33, 425-430; doi: I0.1038/sj.npp. I30 I4I7; published online II April 2007
\end{abstract}

Keywords: antisocial personality disorder; antisocial behavior; MAO-A gene; testosterone; gene by hormone interaction; MHPG

\section{INTRODUCTION}

Antisocial personality disorder (ASPD) and aggression are phenomena that are likely to be caused by a complex interplay of environmental and hereditary factor, and also include behaviors that can be situationally appropriate. ASPD is a diagnosis defined clinically rather than etiologically and includes socially influenced factors such as employment. Nevertheless, ASPD is moderately heritable and cross-transmitted with other dyscontrol disorders (Rhee and Waldman, 2002). ASPD has several well-defined biological correlates including impaired frontal lobe function leading to diminished capacity to inhibit behavior (Dolan and Park, 2002) and decreased levels of the serotonin metabolite 5-HIAA in cerebrospinal fluid (Brown et al, 1979; Roy et al, 1988), reflecting decreased turnover of this inhibitory neurotransmitter in the frontal lobe (Stanley et al, 1985). Aggression is an important manifestation of ASPD, which also displays moderate heritability (Rhee and Waldman, 2002), yet aggression can occur in many healthy individuals and in a manner that falls within the range of normal behavior in certain social situations.

*Correspondence: Dr RL Sjöberg, Laboratory for Neurogenetics, NIH/NIAAA, 5625 Fishers Lane, Rockville, MD 20852, USA, Tel: + I 30 I 443 3242, Fax: + I 30 I 480 2839, E-mail: sjobergr@mail.nih.gov ${ }^{7}$ These authors contributed equally to this work.

Received 13 December 2006; revised 6 March 2007; accepted 9 March 2007
The joint importance of environmental and genetic influences on ASPD is illustrated by the finding that a functional VNTR polymorphism in the monoamine oxidase A (MAOA) gene (Deckert et al, 1999; Sabol et al, 1998) interacts with childhood adversity to predict antisocial personality and antisocial behavior in men (Caspi et al, 2002). The childhood adversity and MAOA interaction has been replicated in at least four studies (Foley et al, 2004; Kim-Cohen et al, 2006; Nilsson et al, 2006; Widom and Brzustowicz, 2006); one study reported a non-significant trend in the same direction (Haberstick et al, 2005) and one study based on a clinical sample has failed to replicate (Young et al, 2006). These studies were all conducted on human males, except Widom and Brzustowicz (2006) which included men and women as will be discussed.

The effect of MAOA genotype on aggressive and antisocial behavior in women appears to be less consistent. In their longitudinal study, Widom and Brzustowicz (2006) found that the combination of childhood abuse and the low activity allele predicted juvenile, but not lifetime violent and antisocial behavior in Caucasian females. Sjoberg et al (2006), studying a sample of 119 adolescent girls (16 and 19 year olds), failed to replicate the effect of MAOA on antisocial behavior visible in the boys they studied (Nilsson et al, 2006) and even described a modest interaction effect in the opposite direction.

There are several reasons why there may be sex differences in the way the MAOA genotype influences antisocial behavior and aggression. One reason is that there 
are considerable sex differences in the prevalence of antisocial personality disorder, with a male preponderance of some 10:1 for the early onset-life course persistent variant (Rutter et al, 2003). Such differences may suggest the operation of sexual selection (Darwin, 1871) leading to differences in the behavioral expression of the same MAOA genotype in males and females. In addition, cultural expectations may reinforce innate differences between the sexes or even create them, leading to differential effects of $M A O A$ genotype.

One possible source of sexual divergence with regard to the MAOA gene is effects of sex hormones such as testosterone. There are several ways in which testosterone may interact with effects of the MAOA gene. First, there is evidence suggesting that both the MAOA gene (MeyerLindenberg et al, 2006)and testosterone (Nef and Parada, 2000) have effects on brain structure and function. Second, and as we explored in the present study, MAOA genotype and testosterone may have an additive or interactive effect on adolescent and adult antisocial behavior. Testosterone has consistently been shown to influence aggression in animal studies and some evidence suggests that it may have similar effects in humans (Archer, 1991; Book et al, 2001). In the sample used in the present study, CSF testosterone levels were higher in individuals with antisocial personality disorder as compared to controls (Virkkunen et al, 1994).

A particular reason to explore $M A O A /$ testosterone interaction is the possibility of direct action of testosterone on the MAOA promoter. The MAOA promoter contains three glucocorticoid/androgen response elements (GRE/ARE) and four binding sites for the Sp1 and R1 transcription factors. Androgens have recently been shown to interact directly with the third GRE/ARE and it also appears to act indirectly with Sp1 (Ou et al, 2006). Although androgens activated $M A O A$ transcription this effect was not as strong as that of glucocorticoids. Since glucocorticoids and testosterone compete for the same sites this may actually mean that high testosterone levels may lead to lower transcription of $M A O A$ and lower MAOA levels. Such an interaction could augment differences between $M A O A$-VNTR alleles.

In vivo studies on human males have furthermore shown that administration of methyl-testosterone negatively influences levels of 3-methoxy-4-hydroxyphenylglycol (MHPG) in cerebrospinal fluid (Daly et al, 2001). Since MHPG is a degradation product of norepinephrine by MAO-A, this finding is consistent with the idea of a direct effect of MAO activity on testosterone. Other recent findings suggesting that cortisol moderates the relationship between testosterone and aggression in delinquent male adolescents (Popma et al, 2006) are also consistent with the molecular mechanisms outlined above. The purpose of the present study was thus to investigate the combined effects of $M A O A$ genotype and testosterone measured in CSF on male ASPD and antisocial behavior.

\section{MATERIALS AND METHODS}

\section{Human Subjects}

The protocol was approved by the Institutional Review Board of the National Institute of Mental Health, the Office for Protection from Research Risks, the University of Helsinki Department of Psychiatry Institutional Review Board, and the University of Helsinki Central Hospital
Institutional Review Board. All subjects provided informed consent before entering the study.

A total of 165 unrelated male criminal alcoholics and controls were collected from the relatively homogeneous Finnish source population. The index cases were 95 criminal alcoholics who, because of the extreme nature of their crimes, were remanded to forensic psychiatric examination at the time of initial incarceration. These subjects spent an average of 1 month on the research ward and were drug- and alcohol-free during this period, and maintained on a low monoamine diet.

The control population consisted of 45 psychiatrically interviewed healthy Finnish male volunteers recruited by advertisements in local newspapers and paid for their participation. They were asked to refrain from use of any alcohol or other drugs and medications for a week before admission, and were then housed on the research ward for 3 days and nights. Both cases and controls were in good general health, as established by physical examination, normal erythrocyte and lymphocyte indexes and thyroid hormone levels, and serum electrolyte and creatinine concentrations. Among cases, $\gamma$-glutamyltransferase levels were also measured in the $48 \mathrm{~h}$ before the start of the study to ascertain alcohol abstinence.

\section{Psychiatric Assessment}

Lifetime psychiatric disorders were diagnosed using the Structured Clinical Interview for DSM (SCID) according to DSM-III-R criteria. Diagnoses were independently blind-rated from interview data by two research psychiatrists under the supervision of a senior research psychiatrist. Inter-rater reliability was very high between the two psychiatrists who blindly rated the interviews. Differences were resolved by the senior psychiatrist, who was also blind to the biochemical data. All cases had either alcohol abuse or alcohol dependence and most $(82 \%)$ were alcohol dependent. Forty eight percent of cases $(N=50)$ met criteria for ASPD. All controls were free from both ASPD and alcohol use disorders.

The Brown-Goodwin Lifetime Aggression Scale was used as a continuous measure of aggression and antisocial behaviors (Brown et al, 1979). This self-report instrument consists of nine questions quantifying, on a four-point scale, aggression towards others (physical as well as verbal), and antisocial behaviors involving disciplinary actions at school or work, with and without police contact.

\section{Cerebrospinal Fluid (CSF) Testosterone}

CSF samples were obtained by lumbar puncture at $0800 \mathrm{~h}$ after bed rest for one night with only water permitted after $2000 \mathrm{~h}$. The samples were collected into a large polypropylene tube on wet ice. After the first $12 \mathrm{ml}$ had been drawn, the tube was capped, inverted, and the CSF sample was aliquoted into $1 \mathrm{ml}$ tubes on dry ice. CSF concentration of free testosterone was measured by radioimmunoassay (Virkkunen et al, 1994).

\section{MAOA (MAOALPR) Genotype}

Genomic DNA was purified from lymphoblastoid cell lines using standard methods from 140 participants, including 95 alcoholics (among whom 43 also had ASPD), and 45 controls. The MAOA promoter region that contains the 
MAOALPR VNTR was amplified from $25 \mathrm{ng}$ genomic DNA using the primer sequences: Forward $5^{\prime}$ CCCAGGCTGCTCC AGAAACATG $3^{\prime}$ and Reverse $5^{\prime}$ GTTCGGGACCTGGGCAGT TGTG-3'. Because of the high GC content in the VNTR region, amplification was performed using Invitrogen's PlatinumTaq and PCRX Enhancer System kits (Invitrogen, Carlsbad, CA), according to the manufacturer's protocol with $5 \mu \mathrm{M}$ of each primer and $25 \mathrm{mM}$ dNTPs in a total reaction volume of $15 \mu \mathrm{l}$. Amplifications were performed on a Perkin Elmer 9700 thermocycler (Applied Biosystems, Foster City, CA) with one cycle at $96^{\circ} \mathrm{C}$ for $10 \mathrm{~min}$ followed by 30 cycles of $94^{\circ} \mathrm{C}$ for $15 \mathrm{~s}, 60^{\circ} \mathrm{C}$ for $15 \mathrm{~s}, 72^{\circ} \mathrm{C}$ for $30 \mathrm{~s}$, and a final $3 \mathrm{~min}$ extension at $72^{\circ} \mathrm{C}$. The forward primer was labeled with the fluorescent dye 6-FAM, and amplicons were visualized on an ABI 3100 capillary sequencer. Allele sizes (allele 3, $263 \mathrm{bp}$; allele 3.5, $278 \mathrm{bp}$; allele 4, $293 \mathrm{bp}$; allele 5, $323 \mathrm{bp}$ ) were determined using Genotyper 2.5 software (Applied Biosystems). The overall success rate for genotyping was $99 \%$ (140/ $141)$. No discrepancies in the duplicates $(N=14)$ were found.

Because the study sample was composed of men only, genotypes were grouped by relative transcriptional activity into two categories: high expression (3.5 or 4 repeats) versus low expression ( 3 or 5 repeats) (Sabol et al, 1998). Because the effect of the five-repeat allele on transcription was inconsistent across studies (Deckert et al, 1999; Sabol et $a l, 1998)$ analyses were repeated excluding the four participants who had the five-repeat genotype.

As reported previously (Ducci et al, 2006) there were no significant differences in $M A O A$ allele frequencies between cases and controls.

\section{Statistical Analyses}

A multiple regression analysis was conducted to test for the effect of three independent variables (MAOALPR genotype, CSF testosterone levels, and the interaction between these two variables (MAOALPR $\times$ testosterone) on the Brown-Goodwin Lifetime Aggression Scale total score. The regression analysis was conducted first by assigning MAOALPR genotype and CSF testosterone levels as independent variables and then as an interaction element. Because main effects in multiple regression models including interaction variables may be influenced by various statistical artefacts, these are not displayed in the results section. Instead we present the main effects from the model that did not include the interaction. Logistic regression was used in a similar way to test the effect of the same independent variables on ASPD diagnosis. CSF testosterone and CSF MPHG levels were compared between ASPD patients and controls using the $t$-test.

A multiple regression analysis was also conducted to test for effect on CSF MHPG of MAOA-LPR, CSF Testosterone and MAOALPR $\times$ testosterone. Also in this case, main effects of $M A O A-L P R$ and testosterone reported refer to those computed before entering the interaction elements.

Other variables that are known to influence CSF monoamine metabolites levels (namely height, BMI, smoking) were entered into the model as independent variables to test for their potential confounding effects. As has been reported elsewhere, the effects of these confounding variables in this sample were significant (Ducci et al, 2006). The correlation between smoking and MHPG was negative and the correlations between BMI and height and
MHPG were positive. Since MAOA also oxidizes serotonine and dopamine, CSF metabolites for these substances (5-HIAA and HVA) were also analyzed in similar ways.

All analyses were conducted using JMP software v5.1 (SAS Institute, Cary, NC). Criterion for statistical significance was set at 0.05 .

\section{RESULTS}

\section{Effect of MAOA Genotype and Testosterone Levels on Antisocial Behavior}

The effects of CSF testosterone and MAOALPR genotype on Brown-Goodwin Lifetime Aggression and ASPD diagnosis are shown in Table 1. As reported previously in this sample (Virkkunen et al, 1994), higher CSF testosterone is significantly associated with an increase in risk of having ASPD (logistic regression, d.f. $=1, \chi^{2}=6.9, P=0.008$ ). CSF testosterone level also tended to be positively correlated with Brown-Goodwin Lifetime Aggression, although this trend was non-significant (linear regression, $\beta=1.27, P=0.09$ ). As reported previously (Ducci et al, 2006), MAOA genotype itself does not predict ASPD in this population (see Table 1).

\section{Interaction of MAOA Genotype and CSF Testosterone on Brown-Goodwin Aggression and ASPD}

Significant interaction effects between $M A O A L P R$ and CSF testosterone were found on both Brown-Goodwin

Table I Relationship of CSF Testosterone and MAOALPR Genotype on Aggression (Brown-Goodwin Lifetime Aggression Scale Total Score), ASPD and CSF MHPG

\begin{tabular}{lccc}
\hline & \multicolumn{3}{c}{ Aggression } \\
\cline { 2 - 4 } Predictors & $\boldsymbol{\beta}(\mathbf{S E})$ & $\boldsymbol{t}$ & $\boldsymbol{P}$ \\
\hline MAOA & $0.74(0.77)$ & 0.96 & 0.33 \\
Testosterone $^{\mathrm{a}}$ & $1.27(0.75)$ & 1.7 & 0.09 \\
MAOA*testosterone & $2.21(0.73)$ & 3.04 & 0.002
\end{tabular}

\begin{tabular}{llll} 
& \multicolumn{3}{c}{ ASPD } \\
\cline { 2 - 4 } & \multicolumn{1}{c}{$\boldsymbol{\beta}(\mathbf{S E})$} & $\boldsymbol{\chi}^{\mathbf{2}}$ & \multicolumn{1}{c}{$\boldsymbol{P}$} \\
\cline { 2 - 4 } MAOA & $0.10(0.23)$ & 0.21 & 0.64 \\
Testosterone $^{\mathrm{a}}$ & $0.67(0.25)$ & 6.9 & 0.008 \\
MAOA $^{*}$ testosterone & $0.61(0.21)$ & 4.5 & 0.04
\end{tabular}

CSF MHPG

\begin{tabular}{lrrr}
\cline { 2 - 4 } MAOA & $-0.64(0.79)$ & -0.81 & 0.03 \\
Testosterone $^{\mathrm{a}}$ & $-1.62(0.77)$ & -2.09 & 0.42 \\
MAOA $^{*}$ testosterone $^{\mathrm{a}}$ & $1.88(0.76)$ & 2.47 & 0.01 \\
\hline
\end{tabular}

ASPD, antisocial personality disorder; $\beta$, regression coefficient; SE, standard error.

${ }^{a}$ CSF testosterone levels have been transformed into z scores (mean of zero and $\mathrm{SD}$ of one) before entry into the model. Therefore, the corresponding $\beta$-value indicates the effect on the outcome variable (eg increase in aggression score or increase in risk of ASPD) for each SD increase in CSF testosterone. 
Lifetime Aggression $(\beta=2.21, \quad P=0.002)$ and ASPD $\left(\chi^{2}=4.5, P=0.02\right)$ (see Table 1$)$. As shown in Figure 1 , higher levels of testosterone were associated with increased Brown-Goodwin scores among individuals with the low activity MAOA genotype (Figure 1a) but not among individuals with the genotype conferring higher levels of MAOA transcription (Figure 1b). Among individuals with the low activity MAOA genotype, a one SD increase in testosterone led to a 3.49 unit increase in aggression score. Consistently, it is only among ASPD patients and controls with the reductionof-function MAOA genotype that there is a case-control difference in CSF testosterone levels. As shown in Figure 2, the case-control comparison for CSF testosterone in individuals with the reduction of function genotype is significant ( $t$-test: d.f. $=1, \mathrm{t}=3.43 ; P=0.001)$. However, there is no difference in CSF testosterone between cases and controls with high $M A O A$ activity genotype ( $t$-test: d.f. $=1 ; \mathrm{t}=0.63, P=0.52$ ).

\section{Interaction of MAOA Genotype and CSF Testosterone on CSF MHPG}

As reported previously (Lappalainen et al, 1999), mean CSF MHPG levels were significantly lower in ASPD (mean $(\mathrm{SE})=35.11(12.43) \mathrm{pmol} / \mathrm{ml}$ ) as compared to controls (mean $(\mathrm{SE})=45.17(12.14) \mathrm{pmol} / \mathrm{ml} ; \mathrm{t}=5.79, P<0.0001) . M A O A-$ LPR genotype were negatively correlated with CSF MHPG $\beta=-0.64 ; P=0.03)$, whereas CSF testosterone did not show any main effect on CSF MHPG (Table 1). Testosterone and $M A O A-L P R$ genotype displayed a significant interaction effect on CSF MHPG $(\beta=1.88 ; P=0.01)$.

As shown in Figure 1, higher levels of testosterone were associated with decreased CSF MHPG among individuals with the reduction-of-function $M A O A$ genotype (Figure 1c) but not among individuals with the genotype conferring higher levels of $M A O A$ transcription (Figure 1d).

The interaction effect between $M A O-L P R$ and testosterone on CSF MHPG remained significant, although it was slightly reduced, after taking into account the effects of BMI, smoking, and height $(\beta=-1.40 ; P=0.05)$.

The MAOA $\times$ testosterone interaction did not predict levels of other CSF metabolites of other MAO substrates (5-HIAA and HVA).

\section{DISCUSSION}

We investigated the joint effects of the functional $M A O A$ VNTR (MAOALPR) and CSF testosterone on ASPD and aggression in human males. As reported previously in this same sample (Virkkunen et al, 1994), individuals with ASPD had higher levels of CSF testosterone but there was no significant predictive effect of MAOALPR genotype alone (Ducci et al, 2006). The main finding of the present study is

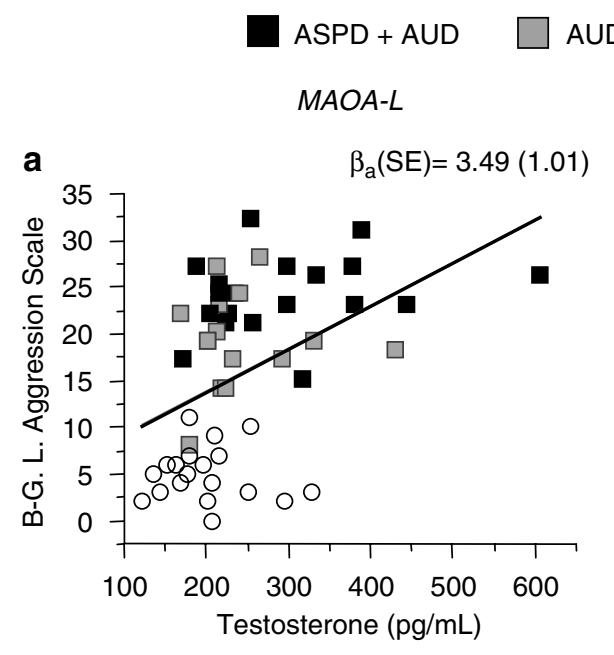

\section{AUD, no ASPD $\bigcirc$ no AUD, no ASPD}
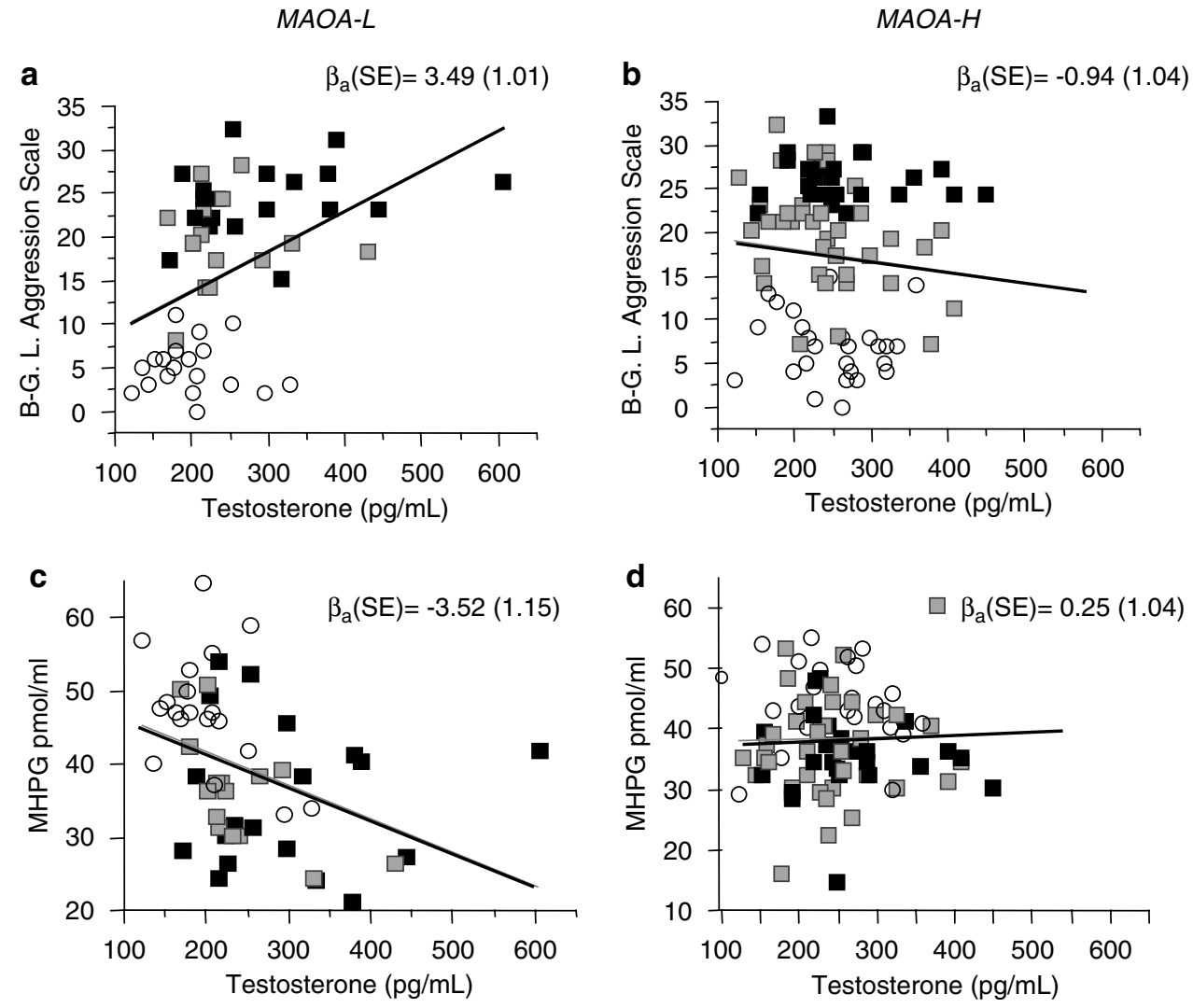

Figure I Correlation between CSF testosterone and aggression in subjects with different MAOA-LPR genotype. MAOA-L = LOW activity (a); MAOA-H = High activity (b). Correlation between CSF testosterone and CSF MHPG in subjects with different MAOA-LPR genotype. MAOA-L=Low activity (c); MAOA-H = High activity (d). ASPD = antisocial personality disorder, AUD: alcohol use disorder; B-G. L. Aggression Scale= Brown-Goodwin lifetime Aggression scale. 

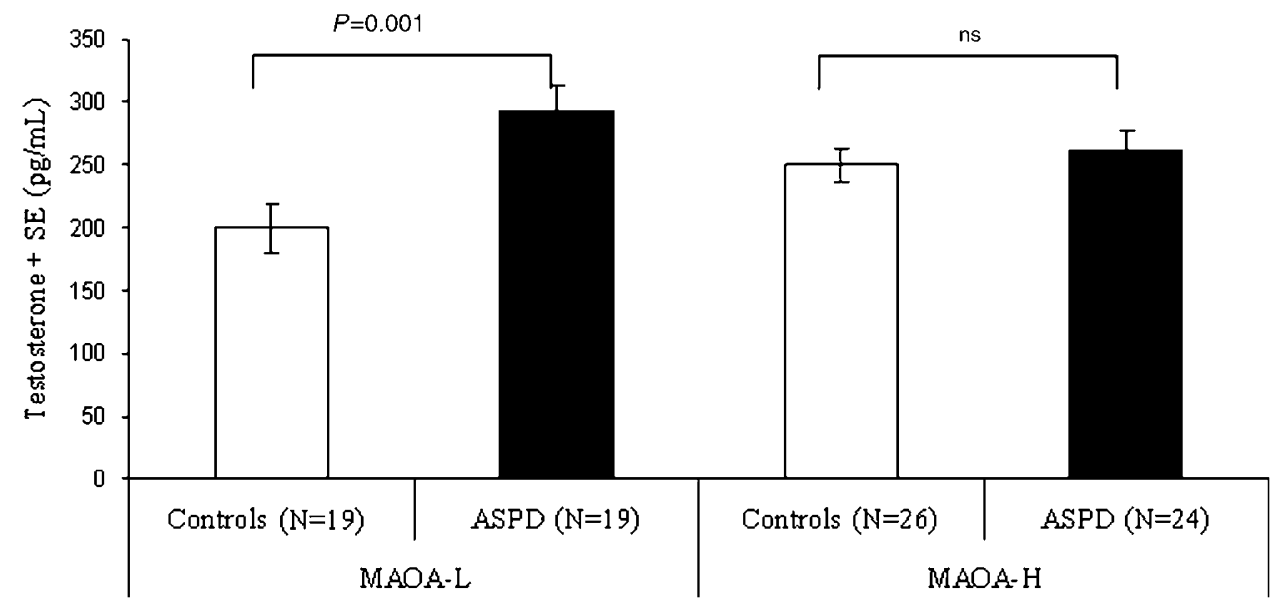

Figure 2 Mean testosterone levels are compared (t-test) between controls (in white) and ASPD patients (in black) among participants with different MAOA-LPR genotypes. MAOA-H = high activity; MAOA-L=low activity. $\mathrm{ns}=$ non significant. $A S P D=$ antisocial personality disorder; $A U D=$ alcohol use disorder; MHPG = 3-methoxy-4-hydroxyphenylglycol.

that testosterone (assessed in CSF) and MAOA genotype interact to predict ASPD as well as lifetime aggression and antisocial behavior measured on the Brown-Goodwin Scale.

There are two possible explanations for our findings. First the MAOA/testosterone interaction may reflect the direct action of testosterone on the MAOA promoter paralleling recently reported in vitro findings (Ou et al, 2006).

The second possibility arises from the functional relationship between brain systems modulated by both MAOA and testosterone, or by either. One recent study positron emission tomography study of brain MAOA activity in relation to $M A O A$-LPR genotype suggests that genotype does not have a direct effect on transcriptional activity (Fowler et al, 2006). This finding may possibly be explained by the fact that the study did not take hormonal and/or environmental factors into account. However, it has been reported that the low-activity MAOA allele predicts amygdala hyperactivity and frontal hypoactivity after an emotional stimulus. Substantial male/female differences in aggression and emotional response are known. For reasons unknown, metabolic responses to emotional stimuli of amygdala and other brain regions differ between men and women (Canli et al, 2002), who apparently have different patterns of emotional modulation. The MAOA enzyme metabolizes dopamine and other monoamine neurotransmitters such as serotonin that are critical in emotional responses and behavioral inhibition. MAO knockout mice exhibit increased aggression and stress reactivity (Shih, 2004), and the low activity $M A O A-L P R$ allele is associated with behavioral dyscontrol and increased aggression. In addition, in one Dutch family, a rare MAOA stop-codon variant leads to behavioral dyscontrol and aggression, further illustrating the power of genetic variation at $M A O A$ to influence this domain of neurobiology (Brunner et al, 1993). The rare MAOA stop-codon variant has been genotyped in the present dataset, but it was not detected (D Goldman et al, unpublished data).

Across studies, testosterone does not consistently increase human aggression despite meta-analyses suggesting that aggression is a behavioral consequence of testosterone. Indeed studies in animal models fairly, consistently show that higher endogenous and administered levels of testosterone predict aggressive behavior (Albert et al, 1993; Archer, 1991; Book et al, 2001). In the human, there are multiple sources of factors that contribute to the variance in aggressive behavior, including differences in previous environmental exposures, variation in testosterone exposure, learning and adaptation, and measurement. However, genetic variation almost certainly plays a role, as established in numerous animal studies. The results of the present study suggest that testosterone response may in part depend on MAOA genotype.

The results also suggests a possible explanation to the fact that previous studies (Sjoberg et al, 2006; Widom and Brzustowicz, 2006) have had difficulties in replicating findings of interactions between environmental adversity and MAOA genotype in females. However, it should be noted that there may be other explanations for this such as a lack of power in these studies. MAOA is an X-linked gene such that about $30 \%$ of men are hemizygous for the low expressing MAOA allele, but only about $9 \%$ of women are homozygous with two copies of the low expressing allele. This suggests that there may have been a relative lack of power to detect effects of MAOA genotype in females. Furthermore the possibility that the $M A O A$-gene escapes $\mathrm{X}$-inactivation in females as suggested by some (Carrel and Willard, 2005) but not all (Nordquist and Oreland, 2006) studies also suggest other possible mechanisms of molecular differences between males and females with respect to this gene.

This study has several strengths including the relative extremity and uniformity of this sample of Finnish offenders and Finnish controls, the fact that our sample consisted entirely of men thus dramatically increasing power to detect effects of the X-linked MAOA gene as well as effects of testosterone found at much higher levels in men, the assessment of ASPD via extensive psychiatric interviews, and the availability of CSF testosterone measures which are more relevant to brain testosterone levels than measures from blood or saliva. However, it should be noted that cause-and-effect inferences for testosterone and behavior in these ASPD subjects are tentative. Although we view this as 
unlikely, higher testosterone levels could be a consequence of the development of ASPD in the context of the low-expressing $M A O A$ genotype. It should also be noted that the results reported in this study were derived from only one, relatively small sample. It is thus important to replicate our findings in other populations before definite conclusions are drawn.

In summary, common, functional $M A O A$ alleles and testosterone interact to predict antisocial behaviors defined as a continuous trait on the Brown-Goodwin scale or categorically via the DSM diagnosis of ASPD. The MAOA/ testosterone interaction also predicted low levels of CSF MHPG, suggesting that the interaction may be mediated by a direct effect on gene transcription. If replicated these findings offer a plausible explanation for previous inconsistencies in studies of the relationship between testosterone and male human aggression, as well as for the understanding of how $M A O A$ genotype may influence behavior.

\section{REFERENCES}

Albert DJ, Walsh ML, Jonik RH (1993). Aggression in humans: what is its biological foundation? Neurosci Biobehav Rev 17: 405-425.

Archer J (1991). The influence of testosterone on human aggression. Br J Psychol 82( Pt 1): 1-28.

Book AS, Starzyk KB, Quinsey VL (2001). The relationship between testosterone and aggression: a meta-analysis. Aggress Violent Behav 6: 579-599.

Brown GL, Goodwin FK, Ballenger JC, Goyer PF, Major LF (1979). Aggression in humans correlates with cerebrospinal fluid amine metabolites. Psychiatry Res 1: 131-139.

Brunner HG, Nelen M, Breakefield XO, Ropers HH, van Oost BA (1993). Abnormal behavior associated with a point mutation in the structural gene for monoamine oxidase A. Science 262: $578-580$.

Canli T, Desmond JE, Zhao Z, Gabrieli JD (2002). Sex differences in the neural basis of emotional memories. Proc Natl Acad Sci USA 99: 10789-10794.

Carrel L, Willard HF (2005). X-inactivation profile reveals extensive variability in $\mathrm{X}$-linked gene expression in females. Nature 434: 400-404.

Caspi A, McClay J, Moffitt TE, Mill J, Martin J, Craig IW et al (2002). Role of genotype in the cycle of violence in maltreated children. Science 297: 851-854.

Daly RC, Su TP, Schmidt PJ, Pickar D, Murphy DL, Rubinow DR (2001). Cerebrospinal fluid and behavioral changes after methyltestosterone administration: preliminary findings. Arch Gen Psychiatry 58: 172-177.

Darwin C (1871). The Descent of Man, and Selection in Relation to Sex. John Murray: London.

Deckert J, Catalano M, Syagailo YV, Bosi M, Okladnova O, Di Bella $\mathrm{D}$ et al (1999). Excess of high activity monoamine oxidase A gene promoter alleles in female patients with panic disorder. Hum Mol Genet 8: 621-624.

Dolan M, Park I (2002). The neuropsychology of antisocial personality disorder. Psychol Med 32: 417-427.

Ducci F, Newman TK, Funt S, Brown GL, Virkkunen M, Goldman D (2006). A functional polymorphism in the MAOA gene promoter (MAOA-LPR) predicts central dopamine function and body mass index. Mol Psychiatry 11: 858-866.

Foley DL, Eaves LJ, Wormley B, Silberg JL, Maes HH, Kuhn J et al (2004). Childhood adversity, monoamine oxidase a genotype, and risk for conduct disorder. Arch Gen Psychiatry 61: 738-744.

Fowler JS, Alia-Klein N, Kriplani A, Logan J, Williams B, Zhu W et al (2006). Evidence that brain MAOA activity does not correspond to MAOA genotype in healthy male subjects. Biol Psychiatry, doi:10.1016/j.biopsych2006.08.038.

Haberstick BC, Lessem JM, Hopfer CJ, Smolen A, Ehringer MA, Timberlake D et al (2005). Monoamine oxidase A (MAOA) and antisocial behaviors in the presence of childhood and adolescent maltreatment. Am J Med Genet B Neuropsychiatr Genet 135: 59-64.

Kim-Cohen J, Caspi A, Taylor A, Williams B, Newcombe R, Craig IW et al (2006). MAOA, maltreatment, and gene-environment interaction predicting children's mental health: new evidence and a meta-analysis. Mol Psychiatry 11: 903-913.

Lappalainen J, Long JC, Virkkunen M, Ozaki N, Goldman D, Linnoila M (1999). HTR2C Cys 23Ser polymorphism in relation to CSF monoamine metabolite concentrations and DSM-III-R psychiatric diagnoses. Biol Psychiatry 46: 821-826.

Meyer-Lindenberg A, Buckholtz JW, Kolachana B, Hariri AR, Pezawas L, Blasi G et al (2006). Neural mechanisms of genetic risk for impulsivity and violence in humans. Proc Natl Acad Sci USA 103: 6269-6274.

Nef S, Parada LF (2000). Hormones in male sexual development. Genes Dev 14: 3075-3086.

Nilsson KW, Sjoberg RL, Damberg M, Leppert J, Ohrvik J, Alm PO et al (2006). Role of monoamine oxidase A genotype and psychosocial factors in male adolescent criminal activity. Biol Psychiatry 59: 121-127.

Nordquist N, Oreland L (2006). Monoallelic expression of MAOA in skin fibroblasts. Biochem Biophys Res Commun 348: 763-767.

Ou XM, Chen K, Shih JC (2006). Glucocorticoid and androgen activation of monoamine oxidase $\mathrm{A}$ is regulated differently by $\mathrm{R} 1$ and Sp1. J Biol Chem 281: 21512-21525.

Popma A, Vermeiren R, Geluk CA, Rinne T, van den Brink W, Knol DL et al (2006). Cortisol Moderates the Relationship between Testosterone and Aggression in Delinquent Male Adolescents. Biol Psychiatry 61: 405-411.

Rhee SH, Waldman ID (2002). Genetic and environmental influences on antisocial behavior: a meta-analysis of twin and adoption studies. Psychol Bull 128: 490-529.

Roy A, Virkkunen M, Linnoila M (1988). Monoamines, glucose metabolism, aggression towards self and others. Int J Neurosci 41: 261-264.

Rutter M, Caspi A, Moffitt TE (2003). Using sex differences in psychopathology to study causal mechanisms: unifying issues and research strategies. J Child Psychol Psychiatry 44: 1092-1115.

Sabol SZ, Hu S, Hamer D (1998). A functional polymorphism in the monoamine oxidase A gene promoter. Hum Genet 103: 273-279.

Shih JC (2004). Cloning, after cloning, knock-out mice, and physiological functions of MAO A and B. Neurotoxicology 25: 21-30.

Sjoberg RL, Nilsson KW, Wargelius HL, Leppert J, Lindstrom L, Oreland L (2006). Adolescent girls and criminal activity: role of MAOA-LPR genotype and psychosocial factors. Am J Med Genet $B$ Neuropsychiatr Genet 144: 159-164.

Stanley M, Traskman-Bendz L, Dorovini-Zis K (1985). Correlations between aminergic metabolites simultaneously obtained from human CSF and brain. Life Sci 37: 1279-1286.

Virkkunen M, Rawlings R, Tokola R, Poland RE, Guidotti A, Nemeroff C et al (1994). CSF biochemistries, glucose metabolism, and diurnal activity rhythms in alcoholic, violent offenders, fire setters, and healthy volunteers. Arch Gen Psychiatry 51: 20-27.

Widom CS, Brzustowicz LM (2006). MAOA and the 'cycle of violence': childhood abuse and neglect, MAOA genotype, and risk for violent and antisocial behavior. Biol Psychiatry 60: 684-689.

Young SE, Smolen A, Hewitt JK, Haberstick BC, Stallings MC, Corley $\mathrm{RD}$ et al (2006). Interaction between MAO-A genotype and maltreatment in the risk for conduct disorder: failure to confirm in adolescent patients. Am J Psychiatry 163: 951-953. 